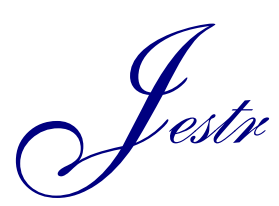

\title{
Performance of Extrinsic Information Scaling Mechanisms with Duo-Binary Turbo Codes
}

\author{
Beeharry Yogesh*, Fowdur Tulsi Pawan and Soyjaudah Krishnaraj M. S. \\ University of Mauritius ,Department of Electrical and Electronic Engineering, Faculty of Engineering, \\ Réduit, Mauritius
}

Received 6 February 2017; Accepted 12 May 2017

\begin{abstract}
Non-binary Turbo codes have gained significant research interest following the adoption of duo-binary Turbo codes in the Digital Video Broadcasting with Return Channel via Satellite (DVB-RCS) standard. In order to meet the Quality of Service (QoS) requirements of applications in communication systems, error control codes with enhanced error performance and low decoding complexity are highly desired. In this paper, the performance analysis of duo-binary Turbo codes using Sign Difference Ratio (SDR) and Regression analysis based extrinsic information scaling mechanisms has been performed. Different techniques for computing the scaling coefficients using the symbol and bit level Log-Likelihood Ratios (LLRs) have been presented and analyzed. Results show that the best gain in error performance is obtained using the mean of scaling coefficients computed from bit-level LLRs with both SDR and Regression based algorithms. The schemes give an average gain of $0.1 \mathrm{~dB}$ as compared to the conventional scheme.
\end{abstract}

Keywords: Duo-binary Turbo codes; SDR; Regression; Scale Factor

\section{Introduction}

Over the decades, Turbo codes [1] have been adopted in several communication standards such as HomePlug Green PHY [5], CDMA 2000 [4], 4G Long Term Evolution (LTE), and Advanced-LTE [2] [3] to enhance transmission over noisy communication channels. However, there is still the need for improvement of error performance with practical packet sizes and reduction in terms of computational complexity with the aim to meet the requirements for applications with stringent delay constraints. Along this line, several techniques have been proposed to improve conventional Turbo decoding algorithms. A lot of research is being conducted on non-binary turbo codes by the Turbo codes community. Duo-binary Turbo codes having a comparable implementational complexity to binary Turbo codes [6], can provide better error correcting performance. The outstanding performance of duo-binary Circular Recursive Systematic Convolutional (CRSC) codes [7] has paved the way to their adoption in the Digital Video Broadcasting with Return Channel via Satellite (DVB-RCS) [8] replacing the conventional scheme of a serial concatenation of a Reed Solomon (RS) code and a convolutional code. The DVB-RCS standard specifies an air-interface consisting of many small terminals connected to a central gateway [9] [10] and sending return signals via satellite. Maximum A-Posteriori Probability (MAP), Logarithmic MAP (Log-MAP) and the Maximum Log-MAP (Max Log-MAP) algorithms are the conventional decoding algorithms for Turbo codes. Due to the extensive numerical

*E-mail address: y.beeharry@uom.ac.mu,

ISSN: 1791-2377 (C) 2017 Eastern Macedonia and Thrace Institute of Technology. All rights reserved. instability and computational complexity of the MAP algorithm, the Log-MAP algorithm [11] has been proposed by researchers. In order to further reduce computational complexity, the Max Log-MAP algorithm was brought forward. This reduction in computational complexity is associated with a slight degradation in error performance as trade-off. An overview of recent schemes for enhancing the error performance of Turbo codes is given next.

In [12], the authors have observed that the extrinsic information output by Max-Log-Maximum-A-posteriori Probability (MAP) Turbo decoders had a negative impact on the a-posteriori probabilities and therefore proposed a scaling factor with a value of less than 1 to attenuate its effect. The typical value which has been reported to achieve good performance is 0.75 [12] when using the IMT2000/3GPP parameters. The authors of [13] have analyzed the effect of the extrinsic information scaling coefficient on the double-iterative decoding algorithm for space-time turbo codes with a large number of antennas. Results have shown that the scaling factors of 0.7 or 0.75 could provide a gain of $0.5 \mathrm{~dB}$ when scaling the extrinsic information in both turbo decoders and at the input of the interference-cancelling block compared to the conventional mechanism without scaling. Furthermore, the work in [14] studied the effect of extrinsic information scaling on Bit Error Rate (BER) and Frame Error Rate (FER) for both single and double binary Turbo codes with MAP and Max Log-MAP decoding algorithms. It has been shown that both decoding algorithms could be enhanced with a scaling factor of $<1$. The authors of [15] have proposed a modified Max Log-MAP Turbo decoding algorithm with an arbitrarily fixed scale factor for the inner decoder and an optimized scale factor for the outer decoder. A mathematical relationship between the scale factor and $\mathrm{E}_{\mathrm{b}} / \mathrm{N}_{0}$ was also proposed. Numerical results 
showed that the proposed scheme can provide a gain of 0.75 $\mathrm{dB}$ over conventional Max Log-MAP decoding at a BER of $2 \times 10^{-5}$ over a Rayleigh fading channel. Building upon the work of [15], the authors of [16] presented a similar mechanism as that of the work in [15] with similar performance for image transmission and retrieval over wireless channel. In [17], the authors proposed a new extrinsic information scaling and early stopping mechanism for long term evolution (LTE) Turbo codes. The scaling factor is obtained by computing the Pearson's correlation coefficient between the extrinsic and a posteriori loglikelihood ratio (LLR) at every half-iteration. The proposed scheme with LTE and QPSK modulation provided a gain of $0.35 \mathrm{~dB}$ on average compared to conventional LTE and QPSK modulation without extrinsic information scaling.

This paper analyses extrinsic information scaling techniques based on Regression analysis [18] and Sign Difference Ratio (SDR) [19] with Duo-Binary Turbo codes. Different techniques for obtaining the scaling coefficients with the symbol-level extrinsic LLRs at the output of each decoder are presented. Schemes with SDR and Regression based extrinsic information scaling using the mean of coefficients obtained from bit-level LLRs provide an average gain of 0.1 $\mathrm{dB}$ over the conventional scheme.

The organisation of the paper is as follows. A background study is presented in Section 2. A detailed description of the proposed system is given in Section 3. Simulation results are presented in Section 4. Section 5 concludes the paper.

\section{System Model}

The system model for non-binary turbo codes with extrinsic information scaling is depicted in Fig. 1. The detailed operation of the model without scaling is explained in [20]. The couples (A, B) of information bits are encoded. The encoder outputs the original systematic information (A and B) together with the parity information (W1, Y1) and (W2, Y2) which correspond to the encoded information pertaining to the upper and lower encoders respectively. These sets of information are then modulated and transmitted through the channel at the transmitter side. At the receiver side, the noisy information is demodulated, de-mapped and sent over to the decoding mechanism. A symbol-level decoding is performed based on the noisy input. The decoding equations and complexity analysis is given and explained in details in [20]. Compared to binary turbo codes, non-binary turbo codes do not have single streams of a-posteriori and extrinsic LLRs. Instead, the decoding mechanism exchanges multiple streams of symbol-level a-posteriori and extrinsic LLRs. The technique used for extrinsic information scaling in this case involves the computation of scale factors using Regression and SDR-based techniques.

The variables shown in the decoding section of Fig. 1 are defined as follows:

$r 0_{t}$ is the received noisy information corresponding to the systematic bits $(\mathrm{A}, \mathrm{B})$ at time instant $t$,

$\overline{r 0}_{t}$ is the interleaved version of $r 0_{t}$,

$r 1_{t}$ is the received noisy information corresponding to the parity bits $(\mathrm{W} 1, \mathrm{Y} 1)$ at time instant $t$,

$r 2_{t}$ is the received noisy information corresponding to the parity bits (W2, Y2) at time instant $t$,

$i \in\{1,2$ or 3$\}$, which is the input symbol,

$n$ is the iteration number, $d \in\{1$ or 2$\}$ which is the decoder number,

$\Lambda_{d e}^{(i, n)}(t)$ is the extrinsic LLR for input symbol $i$ to decoder $d$ at iteration $n$ and time $(t)$,

$\Lambda_{d}^{(i, n)}$ is the a-posteriori LLR for input symbol $i$ to decoder $d$ at iteration $n$ and time $(t)$,

$S_{s d r}^{d(n)}$ is the scale factor based on SDR,

$S_{r e g}^{d(n)}$ is the scale factor based on Regression analysis,

$\hat{A}$ and $\hat{B}$ are the estimated systematic information bits.

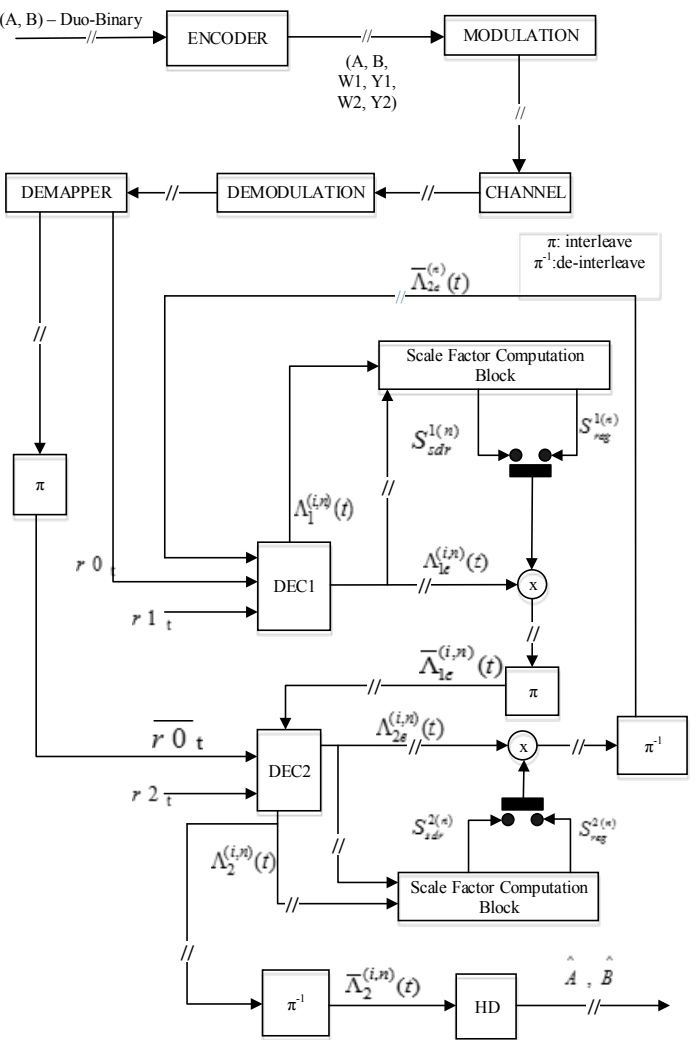

Fig. 1. Complete System Model

The different techniques used for computing the scale factors based on both SDR and Regression are explained next.

\section{A. Scale Factor for each Symbol-Level Extrinsic LLR}

With this technique, a scaling coefficient is computed for each extrinsic LLR using Regression or SDR with each corresponding symbol-level a-posteriori and extrinsic. The 3 different input sets used are: $\left(\Lambda_{d e}^{(1, n)}, \Lambda_{d}^{(1, n)}\right),\left(\Lambda_{d e}^{(2, n)}, \Lambda_{d}^{(2, n)}\right)$, and $\left(\Lambda_{d e}^{(3, n)}, \Lambda_{d}^{(3, n)}\right)$ for the 3 different symbols: 1,2 , and 3 with duo-binary Turbo codes. The equations are as follows:

$S_{s d r, i}^{d(n)}=\frac{1}{N} \sum_{t=1}^{N}\left(f\left(\Lambda_{d e}^{(i, n)}, \Lambda_{d}^{(i, n)}\right)\right)$

$S_{\text {reg,i }}^{d(n)}=\left(\frac{\sum\left(\Lambda_{d e}^{(i, n)}-\bar{\Lambda}_{d e}^{(i, n)}\right) \times\left(\Lambda_{d}^{(i, n)}-\bar{\Lambda}_{d}^{(i, n)}\right)}{\sqrt{\sum\left(\Lambda_{d e}^{(i, n)}-\bar{\Lambda}_{d e}^{(i, n)}\right)^{2} \times \sum\left(\Lambda_{d}^{(i, n)}-\bar{\Lambda}_{d}^{(i, n)}\right)^{2}}}\right)^{2}$

Where,

$S_{s d r, i}^{d(n)}$ is the scale factor based on SDR for symbol $i$,

$f(X, Y)=1$ if $X$ and $Y$ have the same sign, otherwise $f(X, Y)=0$,

$N$ is the couple length, and 
$S_{r e g, i}^{d(n)}$ is the scale factor based on Regression analysis for symbol $i$.

\section{B. Mean Scale Factor from Symbol-Level LLRS}

With this technique, the mean of the 3 different scale factors obtained for each symbol-level extrinsic LLRs is used as the scaling coefficient. The equations are:

$S_{s d r, \text { mean }}^{d(n)}=\frac{1}{3} \sum_{i=1}^{3} S_{s d r, i}^{d(n)}$

$S_{\text {reg,mean }}^{d(n)}=\frac{1}{3} \sum_{i=1}^{3} S_{\text {reg, } i}^{d(n)}$

Where,

$S_{s d r, \text { mean }}^{d(n)}$ and $S_{\text {reg,mean }}^{d(n)}$ are the mean of the 3 different scale factors obtained for each symbol-level extrinsic LLRs using SDR and Regression respectively.

\section{Maximum Scale Factor from Symbol-Level LLRs}

With this technique, the maximum of the 3 different scale factors obtained for each symbol-level extrinsic LLRs is used as the scaling coefficient. The equations are:

$S_{s d r, \max }^{d(n)}=\max _{1 \leq i \leq 3}\left\{S_{s d r, i}^{d(n)}\right\}$

$S_{\text {reg, } \max }^{d(n)}=\max _{1 \leq i \leq 3}\left\{S_{\text {reg, } i}^{d(n)}\right\}$

Where,

$S_{s d r, \text { max }}^{d(n)}$ and $S_{\text {reg,max }}^{d(n)}$ are the maximum of the 3 different scale factors obtained for each symbol-level extrinsic LLRs using SDR and Regression respectively.

\section{Minimum Scale Factor from Symbol-Level LLRs}

With this technique, the minimum of the 3 different scale factors obtained for each symbol-level extrinsic LLRs is used as the scaling coefficient. The equations are:

$S_{s d r, \min }^{d(n)}=\min _{1 \leq i \leq 3}\left\{S_{s d r, i}^{d(n)}\right\}$

$S_{\text {reg, min }}^{d(n)}=\min _{1 \leq i \leq 3}\left\{S_{\text {reg, } i}^{d(n)}\right\}$

Where,

$S_{s d r, \min }^{d(n)}$ and $S_{r e g, \min }^{d(n)}$ are the minimum of the 3 different scale factors obtained for each symbol-level extrinsic LLRs using SDR and Regression respectively.

\section{E. Mean Scale Factor from Bit-Level LLRs}

With this technique, the bit-level a-posteriori and extrinsic LLRs are first approximated from the corresponding symbol-level LLRs. Table 1 shows the bit representations of the symbols in duo-binary Turbo codes.

The equations for the approximate bit-level a-posteriori and extrinsic LLRs can be obtained as follows (Shan, Lei, Huifang, \& Kuang, 2010):

$$
\Lambda_{d e}^{\left(b_{0}, n\right)}=\max \left(\Lambda_{d e}^{(2, n)}, \Lambda_{d e}^{(3, n)}\right)-\max \left(\Lambda_{d e}^{(0, n)}, \Lambda_{d e}^{(1, n)}\right)
$$

$$
\begin{aligned}
& \Lambda_{d e}^{\left(b_{1}, n\right)}=\max \left(\Lambda_{d e}^{(1, n)}, \Lambda_{d e}^{(3, n)}\right)-\max \left(\Lambda_{d e}^{(0, n)}, \Lambda_{d e}^{(2, n)}\right) \\
& \Lambda_{d}^{\left(b_{0}, n\right)}=\max \left(\Lambda_{d}^{(2, n)}, \Lambda_{d}^{(3, n)}\right)-\max \left(\Lambda_{d}^{(0, n)}, \Lambda_{d}^{(1, n)}\right) \\
& \Lambda_{d}^{\left(b_{1}, n\right)}=\max \left(\Lambda_{d}^{(1, n)}, \Lambda_{d}^{(3, n)}\right)-\max \left(\Lambda_{d}^{(0, n)}, \Lambda_{d}^{(2, n)}\right)
\end{aligned}
$$

Table 1. Bit Representation of symbols in Duo-Binary Turbo codes.

\begin{tabular}{c|c|c}
\hline Symbol & $\boldsymbol{b}_{\mathbf{0}}$ & $\boldsymbol{b}_{\mathbf{1}}$ \\
\hline 0 & 0 & 0 \\
1 & 0 & 1 \\
2 & 1 & 0 \\
3 & 1 & 1 \\
\hline
\end{tabular}

Where,

$\Lambda_{d e}^{\left(b_{0}, n\right)}$ is the bit-level extrinsic LLR for the first position $b_{0}$, $\Lambda_{d e}^{\left(b_{1}, n\right)}$ is the bit-level extrinsic LLR for the second position $b_{1}$,

$\Lambda_{d}^{\left(b_{0}, n\right)}$ is the bit-level a-posteriori LLR for the first position $b_{0}$,

$\Lambda_{d}^{\left(b_{1}, n\right)}$ is the bit-level a-posteriori LLR for the first position $b_{1}$.

The 2 different input sets used in this case are: $\left(\Lambda_{d e}^{\left(b_{0}, n\right)}, \Lambda_{d}^{\left(b_{0}, n\right)}\right), \quad$ and $\quad\left(\Lambda_{d e}^{\left(b_{1}, n\right)}, \Lambda_{d}^{\left(b_{1}, n\right)}\right)$. The corresponding scaling coefficients for both

\section{F. Max Scale Factor from Bit-Level LLRs}

With this technique, the maximum of the 2 scale coefficients obtained from the bit-level LLR approximations is used to scale the 3 symbol-level extrinsic LLRs. The equations are:

$S_{s d r, b, \max }^{d(n)}=\max _{0 \leq x \leq 1}\left\{S_{s d r, b_{x}}^{d(n)}\right\}$

$S_{\text {reg,b,max }}^{d(n)}=\max _{0 \leq x \leq 1}\left\{S_{\text {reg, } b_{x}}^{d(n)}\right\}$

\section{G. Min Scale Factor from Bit-Level LLRs}

With this technique, the minimum of the 2 scale coefficients obtained from the bit-level LLR approximations is used to scale the 3 symbol-level extrinsic LLRs. The equations are:

$S_{s d r, b, \min }^{d(n)}=\min _{0 \leq x \leq 1}\left\{S_{s d r, b_{x}}^{d(n)}\right\}$

$S_{\text {reg,b,min }}^{d(n)}=\min _{0 \leq x \leq 1}\left\{S_{\text {reg, } b_{x}}^{d(n)}\right\}$

\section{Simulation Results}

The analysis of the following schemes in terms of BER performance has been made:

Scheme 1 - Conventional duo-binary Turbo decoding.

Scheme S1 - Duo-binary Turbo decoding with 3 different SDR-based extrinsic information scale factors obtained using symbol-level LLRs.

Scheme S2 - Duo-binary Turbo decoding with the mean of 3 different SDR-based extrinsic information scale factors obtained using symbol-level LLRs. 
Scheme S3 - Duo-binary Turbo decoding with the maximum of 3 different SDR-based extrinsic information scale factors obtained using symbol-level LLRs.

Scheme S4 - Duo-binary Turbo decoding with the minimum of 3 different SDR-based extrinsic information scale factors obtained using symbol-level LLRs.

Scheme S5 - Duo-binary Turbo decoding with the mean of 2 different SDR-based extrinsic information scale factors obtained using bit-level LLRs.

Scheme S6 - Duo-binary Turbo decoding with the max of 2 different SDR-based extrinsic information scale factors obtained using bit-level LLRs.

Scheme S7 - Duo-binary Turbo decoding with the min of 2 different SDR-based extrinsic information scale factors obtained using bit-level LLRs.

Scheme R1 - Duo-binary Turbo decoding with 3 different Regression-based extrinsic information scale factors obtained using symbol-level LLRs.

Scheme R2 - Duo-binary Turbo decoding with the mean of 3 different Regression-based extrinsic information scale factors obtained using symbol-level LLRs.

Scheme R3 - Duo-binary Turbo decoding with the maximum of 3 different Regression-based extrinsic information scale factors obtained using symbol-level LLRs. Scheme R4 - Duo-binary Turbo decoding with the minimum of 3 different Regression-based extrinsic information scale factors obtained using symbol-level LLRs.

Scheme R5 - Duo-binary Turbo decoding with the mean of 2 different Regression-based extrinsic information scale factors obtained using bit-level LLRs.

Scheme R6 - Duo-binary Turbo decoding with the maximum of 2 different Regression-based extrinsic information scale factors obtained using bit-level LLRs.

Scheme R7 - Duo-binary Turbo decoding with the minimum of 2 different Regression-based extrinsic information scale factors obtained using bit-level LLRs.

Simulations were performed using a duo-binary Turbo codes with the following parameters:

Couple-length of 212 ,

5000 packets,

Code-rate of $1 / 3$,

QPSK modulation, and

Complex Additive White Gaussian Noise (AWGN) Channel.

The BER performances for duo-binary Turbo codes with the different SDR-based extrinsic information scaling mechanisms are depicted in Fig. 2.Fig. 2 shows the BER performances of the schemes with SDR-based extrinsic information scaling. It can be observed that all schemes with SDR-based scaling perform better than the conventional Scheme 1. Scheme S5 performs better than all other schemes in the error-floor region. The BER performances for duobinary Turbo codes with the different Regression-based extrinsic information scaling mechanisms are depicted in Fig. 3. Fig. 3 shows the BER performances of the schemes with Regression-based extrinsic information scaling. It can be observed that all schemes with Regression-based scaling perform better than the conventional Scheme 1. Scheme R5 performs better than all other schemes in the error-floor region. The BER performances for duo-binary Turbo codes with the best SDR and Regression-based extrinsic information scaling mechanisms are depicted in Fig. 4. Fig. 4 shows that with both SDR and Regression based extrinsic information scaling mechanisms, Schemes S5 and R5 which use the mean of the coefficients computed using bit-level LLRs, have almost similar error performances. Both Schemes S5 and R5 give an average gain of approximately $0.1 \mathrm{~dB}$ compared to the conventional scheme without extrinsic information scaling.

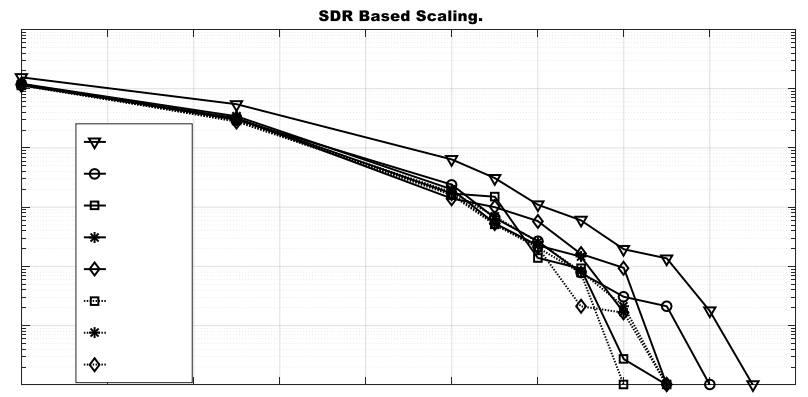

Fig. 2. BER Performance with different SDR-based extrinsic information scaling mechanisms

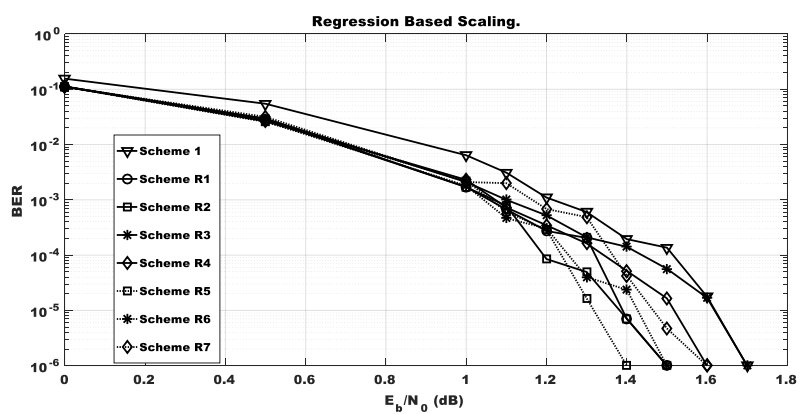

Fig. 3. BER Performance with different Regression-based extrinsic information scaling mechanisms

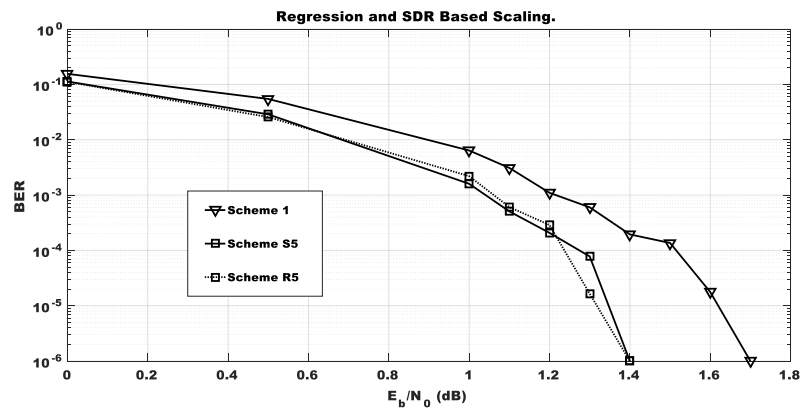

Fig. 4. BER Performance with the best SDR and Regression-based extrinsic information scaling mechanisms

\section{Conclusions}

In this paper, an analysis of duo-binary Turbo codes using SDR and Regression analysis based extrinsic information scaling mechanisms has been performed. Different techniques for computing the scaling coefficients using the symbol and bit-level LLRs have been presented and analyzed. Results demonstrate that the best error performances are obtained using the mean of scaling coefficients computed from bit-level LLRs and both SDR and Regression based algorithms. These schemes give an average approximate gain of $0.1 \mathrm{~dB}$ compared to the conventional scheme without extrinsic information scaling.

However, the schemes for extrinsic information scaling add considerable computational complexity in the decoding mechanism. As such, techniques for early stopping would be required to halt the decoding process such that no unnecessary additional iterations are performed. The stopping mechanisms employing SDR and Regression based 
techniques as presented in the literature with binary Turbo codes cannot be directly extrapolated to non-binary Turbo codes. An interesting future work would thus be to search for the appropriate stopping mechanisms for non-binary Turbo codes using Regression based analysis.

\section{Acknowledgements}

The technical support of the University of Mauritius and the Tertiary Education Commission of Mauritius is duly acknowledged.
This is an Open Access article distributed under the terms of the Creative Commons Attribution Licence

\section{References}

[1] C. Berrou, A. Glavieux and P. Thitimajshima, "Near Shannon limit error-correcting coding and decoding," in IEEE International Conference on Communications, ICC 93, Geneva, 1993.

[2] S. Seisa, I. Toufik and M. Baker, LTE - The UMTS Long Term Evolution: From Theory to Practice, John Wiley and Sons Ltd, 2009.

[3] "3GPP: Technical Specifications Rel. 8," 3GPP, 2009.

[4] "3GPP2 C.S0024-B: 'CDMA 2000 High Rate Packet Data Air Interface Specification' Version 1.0," May 2006. [Online]. Available: http://www.3gpp2.org/Public_html/specs/C.S0024 B v1.0 060522.pdf. [Accessed November 2006].

[5] J. Zyren, "Home Plug Green PHY Overview, Technical Paper," Atheros Communications, 2010.

[6] C. Berrou and M. Jézéquel, "Non Binary Convolutional Codes for Turbo Coding," IEEE Electronic Letters, vol. 35, no. 1, pp. 39 40, 1999.

[7] C. Berrou, C. Douillard and M. Jézéquel, "Multiple Parallel Concatenation of Circular Recursive Convolutional (CRSC) codes," Annals of Telecommunications, vol. 54, no. 3 - 4, pp. 166 - 172, 1999.

[8] D. Giancristofaro and A. Bartolazzi, "Novel DVB-RCS standard turbo codes: details and performance of a decoding algorithm," Seventh International Workshop in Digital Signal Processing Techniques for Space Communications, Oct. 2001.

[9] M. R. Soleymani, Y. Gao and U. Vilaipornsawai, Turbo Coding for Satellite and Wireless Communications, New York: Kluwer Academic Publishers, 2002.

[10] M. R. Soleymani and Y. Gao, "Spectrally efficient non-binary turbo codes: Beyond DVB-RCS standard," Proceedings of 21st Biennial Symposium on Communications, vol. 3, pp. 951-955, May 2002.

[11] T. Park, M. Kim, C. Kim and J. Jung, "Analysis of Turbo codes for Next Generation DVB-RCS system," in 28th American Institute of Aeronautics and Astronautics International Communications Satellite Systems Conference (ICSSC), Anaheim, California, 2010

[12] J. Vogt and A. Finger, "Improving the MAX-Log-MAP Turbo decoder," Electr. Lett., vol. 36, no. 23, pp. 1937 - 1939, 2000.
[13] L. Trifina, D. Tarniceriu and A. M. Rotopanescu, "Influence of Entrinsic Information Scaling Coefficient on Double-Iterative Decoding Algorithm for Space Time Turbo Codes with Large Number of of Antennas," Adv. Electr. Comput. Eng., vol. 11, no. 1, pp. 85 - 90, 2011

[14] H. Balta and C. Douillard, "On the Influence of the Extrinsic Information Scaling Coefficient on the Performance of Single and Double Binary Turbo Codes," Adv. Electr. Comput. Eng., vol. 13, no. 2, pp. $77-84,2013$.

[15] R. Krishnamoorthy and N. S. Pradeep, "Modified Max-Log-MAP turbo decoding algorithm using optimised scale factor," in International Conference on Recent Trends in Information Technology, 2013.

[16] M. B. Moses and N. S. Pradeep, "Performance improvement of Max-Log-MAP Turbo decoding algorithm using optimized scaling factor for image transmission and retrieval," in International Conference on Green Computing and Internet of Things, 2015

[17] T. P. Fowdur, Y. Beeharry and K. M. S. Soyjaudah, "A novel scaling and early stopping mechanism for LTE turbo code based on regression analysis," Annals of Telecommunications, vol. 71, no. 7, pp. 369 - 388, 2016.

[18] R. Lowry, "Concepts and Applications of Inferential Statistics," 1998 - 2013. [Online]. Available: http://vassarstats.net/textbook/. [Accessed 24 November 2014].

[19] Y. Lin, W. Humg, W. Lin, T. Chen and E. Lu, "An efficient SoftInput Scaling Schene for Turbo Decoding," in IEEE International Conference on Sensor Networks, Ubiquitous, and Trustworthy Computing Workshops, 2006.

[20] Y. Beeharry, T. P. Fowdur and K. M. S. Sojaudah, "SymbolLevel Decoding Algorithms for Duo-Binary Turbo Codes," IIUM Journal (In Press), 2017.

[21] L. Shan, X. Lei, C. Huifang and W. Kuang, "A New stopping criteria for duo-binary turbo codes," in International Conference on Communications anf Mobile Computing (CMC), Shenzen, 2010. 